\title{
Patterns of care in hilar node-positive (N1) non-small cell lung cancer: A missed treatment opportunity?
}

\author{
Matthew J. Bott, MD, ${ }^{a}$ Aalok P. Patel, BS, ${ }^{a}$ Vivek Verma, MD, ${ }^{\mathrm{b}}$ Traves D. Crabtree, MD, ${ }^{\mathrm{a}}$ \\ Daniel Morgensztern, MD, ${ }^{\mathrm{c}}$ Clifford G. Robinson, MD, ${ }^{\mathrm{d}}$ Graham A. Colditz, PhD, ${ }^{\mathrm{a}}$ Saiama Waqar, MD, \\ Daniel Kreisel, MD, PhD, ${ }^{\mathrm{a}}$ A. Sasha Krupnick, MD, ${ }^{\mathrm{a}}$ G. Alexander Patterson, MD, ${ }^{\mathrm{a}}$ \\ Stephen Broderick, MD, ${ }^{\mathrm{a}}$ Bryan F. Meyers, MD, ${ }^{\mathrm{a}}$ and Varun Puri, MD, MSCI ${ }^{\mathrm{a}}$
}

\begin{abstract}
Background: For patients with non-small cell lung cancer (NSCLC) metastatic to hilar lymph nodes (N1), guidelines recommend surgery and adjuvant chemotherapy in operable patients and chemoradiation (CRT) for those deemed inoperable. It is unclear how these recommendations are applied nationally, however.
\end{abstract}

Methods: The National Cancer Database was queried to identify patients with a tumor $<7 \mathrm{~cm}$ (T1/T2) with clinically positive N1 nodes. Patients undergoing CRT (comprising chemotherapy and radiation $>45 \mathrm{~Gy}$ ) or surgical resection were considered adequately treated. Remaining patients were classified as receiving inadequate or no treatment.

Results: Of the 20,366 patients who met the study criteria, $63 \%$ underwent adequate treatment (48\% surgical resection, $15 \%$ CRT). The remainder received inadequate treatment $(23 \%)$ or no treatment $(14 \%)$. In univariate analysis, the patients receiving inadequate or no treatment were older, tended to be non-Caucasian, had a lower income, and had a higher comorbidity score. Patients undergoing adequate treatment had improved overall survival (OS) compared with those receiving inadequate or no treatment (median OS, 34.0 months vs 11.7 months; $P<.001$ ). Of those receiving adequate treatment, logistic regression identified several variables associated with surgical resection, including treatment at an academic facility, Caucasian race, and annual income $>\$ 35,000$. Increasing age and T2 stage were associated with nonoperative management. Following propensity score matching of 2308 patient pairs undergoing surgery or CRT, resection was associated with longer median OS (34.1 months vs 22.0 months; $P<.001)$.

Conclusions: Despite the established guidelines, many patients with T1-2N1 NSCLC do not receive adequate treatment. Surgery is associated with prolonged survival in selected patients. Surgical input in the multidisciplinary evaluation of these patients should be mandatory. (J Thorac Cardiovasc Surg 2016;151:1549-58)

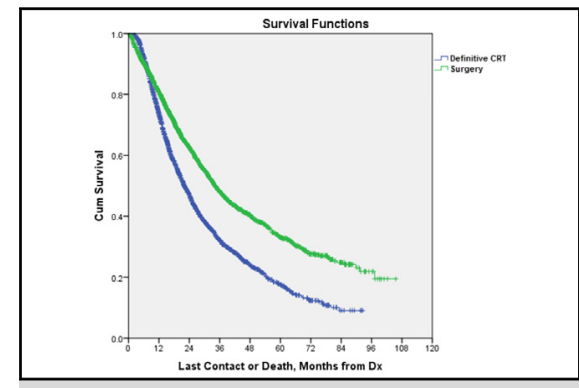

Propensity-matched survival for patients undergoing surgery versus CRT for N1-positive NSCLC.

\section{Central Message}

Despite the existence of published guidelines, many patients with T1-2N1 NSCLC do not receive adequate treatment. Surgical evaluation should be mandatory in these patients.

\section{Perspective}

Published guidelines recommend surgery as the primary treatment modality for operable patients with hilar node-positive (N1) NSCLC and chemoradiation (CRT) for those deemed inoperable. Our analysis of a large national database shows that adherence to these guidelines is poor. We show that $<50 \%$ of T1-2N1 patients undergo resection, and that many selected for CRT receive inadequate therapy.

See Editorial Commentary page 1559 .

\footnotetext{
From the ${ }^{\mathrm{a}}$ Division of Cardiothoracic Surgery, Department of Surgery, ${ }^{\mathrm{c}}$ Division of Oncology, Department of Medicine, and ${ }^{\mathrm{d}}$ Department of Radiation Oncology, Washington University School of Medicine, St Louis, Mo; 'bepartment of Radiation Oncology, University of Nebraska Medical Center, Omaha, Neb.

Varun Puri is supported by National Institutes of Health Grants K07CA178120 and K12CA167540-02 (Paul Calabresi Award).

Read at the 41st Annual Meeting of the Western Thoracic Surgical Association, Whistler, British Columbia, Canada, June 25, 2015.

Received for publication June 25, 2015; revisions received Dec 17, 2015; accepted for publication Jan 27, 2016.

Address for reprints: Varun Puri, MD, MSCI, Division of Cardiothoracic Surgery, Washington University School of Medicine, Campus Box 8234, 660 S Euclid Ave, St Louis, MO 63110 (E-mail: puriv@wudosis.wustl.edu). $0022-5223 / \$ 36.00$

Copyright (c) 2016 by The American Association for Thoracic Surgery http://dx.doi.org/10.1016/j.jtcvs.2016.01.058
}

Node-positive non-small cell lung cancer (NSCLC) is an aggressive disease with high mortality ${ }^{1}$; however, patients with disease limited to pulmonary and hilar lymph nodes (N1) may experience long-term survival with aggressive, multimodal therapy. ${ }^{2}$ In patients with acceptable operative

Scanning this QR code will take you to supplemental tables for this article.

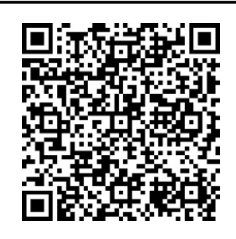




\section{Abbreviations and Acronyms \\ AJCC = American Joint Committee on Cancer \\ $\mathrm{CI}=$ confidence interval \\ $\mathrm{CRT}=$ chemoradiation \\ $\mathrm{CT}=$ computed tomography \\ $\mathrm{NCDB}=$ National Cancer Database \\ NSCLC $=$ non-small cell lung cancer \\ OR $=$ odds ratio \\ OS = overall survival \\ PET = positron emission tomography}

risk, surgical resection with adjuvant chemotherapy forms the cornerstone of treatment for $\mathrm{N} 1$ disease. ${ }^{3}$ Although studies directly evaluating treatment of medically inoperable patients with $\mathrm{N} 1$ disease are lacking, extrapolation of data from stage III patients suggests that chemoradiation (CRT) is generally the preferred standard of care..$^{4-7}$ Despite the existence of established guidelines outlining these treatment paradigms, adherence to these recommendations at the national level is unclear.

The National Cancer Database (NCDB) is a joint program developed in 1989 by the Commission on Cancer, the American College of Surgeons, and the American Cancer Society. ${ }^{8}$ The database contains data submitted by more than 1500 accredited cancer centers across the United States and Puerto Rico, and it captures approximately $70 \%$ of all new cancer cases diagnosed in the United States annually. To better characterize the treatment of N1 disease nationwide, we queried the NCDB to examine patterns of care regarding N1 (T1 or T2) NSCLC in the United States. We hypothesized that despite the existence of established guidelines, physician practice and surgical referral for this disease would vary considerably.

\section{METHODS}

We queried the NCDB to identify patients treated for clinical N1 node-positive NSCLC (hilar, interlobar, lobar, or segmental nodes) between 1998 and 2010.9 All information was deidentified, and so the need for Institutional Review Board approval for the study was waived by Washington University. The analysis was limited to patients with T1 or T2 disease (generally representing stage II NSCLC according to the American Joint Committee on Cancer [AJCC] staging manual, 7th edition). ${ }^{10}$ Those patients with clinical $\mathrm{T} 3$ or $\mathrm{T} 4$ tumors, or those with clinically positive mediastinal lymph nodes (N2 disease) were specifically excluded. Patients undergoing either surgical resection or CRT with $>45$ Gy of radiation were considered adequately treated. Chemotherapy and radiation could be given in any order. Patients not meeting these treatment criteria were classified as receiving inadequate (ie, some chemotherapy and/or radiation but not meeting the previously defined threshold for adequate therapy) or no treatment.

Information regarding patient- and tumor-related variables, treatment details, and short- and long-term outcomes was extracted. Using information on race, income, and population size of the area from which a patient presented, we created dichotomized groups in which a patient was either Caucasian or not Caucasian, had an annual income $<\$ 35,000$ or $>\$ 35,000$, and presented from a rural location (regional population $<250,000$ ) or an urban location, respectively. The Charlson/ Deyo score was used as a measure of comorbidity; possible scores were 0,1 , or $\geq 2$. Because very few patients have a score $>2$, the NCDB combines patients with scores of $\geq 2$ into a single group. Treatment facilities were classified as community cancer programs, comprehensive community cancer programs, or academic/research centers. For the analysis, community cancer programs and comprehensive community cancer programs were combined in the category of nonacademic centers.

Last known vital status and the time between the date of diagnosis and the date of follow-up were used to determine survival. The NCDB defines the date of diagnosis as the date of histological confirmation of NSCLC in cases for which that information is available. In cases where the diagnosis was made based on imaging and the patient proceeded directly to resection without biopsy, the date of diagnosis is defined as the date of the radiologic imaging that identified the lesion.

All analyses were performed using SPSS 21.0 for Windows (IBM, Armonk, NY). Descriptive statistics are expressed as mean \pm standard deviation unless specified otherwise. Independent-samples $t$ tests and 1-way analysis of variance were used to compare continuous variables. The $\chi^{2}$ test was used to compare categorical data. Overall survival (OS) was estimated by the Kaplan-Meier method. Multivariate logistic regression models were fitted to evaluate variables associated with surgical resection. Factors accounted for in the multivariate analysis included age, tumor size, sex, race, facility type (academic vs nonacademic), income, urban location, Charlson/Deyo score, and clinical T stage.

Propensity score matching was performed to identify 2 equivalent cohorts of patients either undergoing surgery or receiving adequate CRT. The propensity score was the probability of receiving CRT during the study period, estimated using a logistic regression model that included age, race, sex, income, facility type (academic vs nonacademic), Charlson/Deyo score, urban location, clinical $\mathrm{T}$ stage, and tumor size. These variables were selected from an initial univariate analysis comparing the surgery and chemotherapy groups, and variables that differed significantly between the 2 groups were chosen for propensity matching. Patients for whom the propensity scores matched to the fourth decimal place were matched in 1:1 fashion. Automated matching was performed using the Fuzzy extension command in SPSS 21.0. For all analyses, a $P$ value $<.05$ was considered statistically significant.

\section{RESULTS}

A total of 20,366 patients who met the study criteria were identified in the NCDB between 1998 and 2010. These patients were all noted to have NSCLC with clinically positive N1 lymph nodes. Of these, 12,857 patients $(63 \%)$ received adequate treatment as defined in Methods (surgical resection, $\mathrm{n}=9719$ [48\%]; definitive CRT, $\mathrm{n}=3138$ $[15 \%])$. The remaining 7509 patients $(37 \%)$ received either inadequate CRT $(\mathrm{n}=4640 ; 23 \%)$ or no treatment $(\mathrm{n}=2869 ; 14 \%)$.

Table 1 compares demographic data and tumor- and treatment-related information in patients receiving adequate therapy and those receiving inadequate or no therapy. In the univariate analyses, compared with the patients receiving adequate treatment, those receiving inadequate or no treatment were older, more likely to be non-Caucasian, had a lower annual income, and had a higher Charlson/Deyo comorbidity score. These patients also were more likely to have slightly larger tumors, as 
TABLE 1. Demographic data and clinical characteristics of patients with clinical N1-positive NSCLC receiving adequate therapy and those receiving inadequate or no therapy

\begin{tabular}{|c|c|c|c|}
\hline Patient characteristic & Inadequate or no treatment $(n=7509)$ & Surgery or adequate CRT $(n=12,857)$ & $P$ value \\
\hline Age at diagnosis, $\mathrm{y}$, mean $\pm \mathrm{SD}$ & $72.7 \pm 10.1$ & $66.9 \pm 10.0$ & $<.001$ \\
\hline Male sex, n (\%) & $4059(54)$ & $7099(55)$ & .11 \\
\hline Caucasian race, $\mathrm{n}(\%)$ & $6458(86)$ & $11,328(88)$ & $<.001$ \\
\hline Academic center, n (\%) & $1728(23)$ & $3928(31)$ & $<.001$ \\
\hline Annual income $>\$ 35,000, \mathrm{n}(\%)$ & $4353(61)$ & $7880(65)$ & $<.001$ \\
\hline Urban population area, $\mathrm{n}(\%)$ & $4735(63)$ & $7955(62)$ & .09 \\
\hline \multicolumn{4}{|l|}{ Charlson/Deyo score, n (\%) } \\
\hline 0 & $4401(59)$ & $7275(57)$ & \multirow[t]{3}{*}{$<.001$} \\
\hline 1 & $2073(28)$ & $4121(32)$ & \\
\hline 2 & $1035(14)$ & $1461(11)$ & \\
\hline Tumor size, mm, mean \pm SD & $41.1 \pm 31.9$ & $39.8 \pm 35.1$ & .01 \\
\hline \multicolumn{4}{|l|}{ AJCC clinical T stage, $\mathrm{n}(\%)$} \\
\hline 1 & $2296(31)$ & $4629(36)$ & \multirow[t]{2}{*}{$<.001$} \\
\hline 2 & $5213(69)$ & $8228(64)$ & \\
\hline \multicolumn{4}{|l|}{ AJCC clinical stage group, $\mathrm{n}(\%)$} \\
\hline $2 \mathrm{~A}$ & $2517(33)$ & $5322(41)$ & \multirow[t]{3}{*}{$<.001$} \\
\hline $2 \mathrm{~B}$ & $4658(62)$ & $7143(56)$ & \\
\hline 2 NOS & $334(4)$ & $392(3)$ & \\
\hline Any radiotherapy, $\mathrm{n}(\%)$ & $3342(44)$ & $4910(38)$ & $<.001$ \\
\hline Total radiation dose, $c \mathrm{~Gy}$, mean $\pm \mathrm{SD}$ & $5334 \pm 3264$ & $6284 \pm 3427$ & $<.001$ \\
\hline Any chemotherapy, n (\%) & $2241(30)$ & $8244(64)$ & $<.001$ \\
\hline
\end{tabular}

CRT, Chemoradiation; SD, standard deviation; AJCC, American Joint Committee on Cancer; NOS, not otherwise specified.

reflected by increased $\mathrm{T}$ stage and a higher incidence of stage IIB versus IIA disease. Among the patients who received treatment but failed to meet the standard for adequate therapy, $72 \%$ received at least some radiation, whereas only $48 \%$ received any chemotherapy (34\% received a multiagent regimen). Neoadjuvant therapy was infrequent, with only $6 \%$ of patients receiving induction chemotherapy and $4 \%$ receiving preoperative radiation. The use of adjuvant radiation was more common in patients with a positive pathological margin ( $42 \%$ vs $15 \%$ in those with a negative margin; $P<.001)$.

Results from Kaplan-Meier analysis of OS in patients undergoing various treatments are shown in Figure 1 and Tables E1 and E2. Patients either undergoing surgical resection or receiving adequate CRT had prolonged OS compared with those receiving inadequate or no treatment (median OS, 34.0 months [95\% CI, 32.8-35.1 months] vs 11.7 months [95\% CI, 11.4-12.1 months); $P<.001$ ] (Figure 1, $A$ and Table E1). In addition, inadequate treatment was associated with longer OS compared with no therapy (median OS, 13.7 months $[95 \%$ CI, 13.2-14.2 months] vs 8.1 months [ $95 \%$ CI, 7.6-8.6 months]; $P<.001$ ) (Figure 1, $B$ and Table E2).

To investigate the variation in these treatment approaches over time, we divided the patient sample into 2 cohorts based on treatment date (2003-2006 and 2007-2010). Patients treated during the more recent time period (2007-2010) were significantly more likely to receive adequate treatment ( $70 \%$ vs $54 \%)$.
Demographic and clinical characteristics of patients undergoing surgery and those receiving definitive CRT are compared in Table 2. On univariate analyses, patients undergoing surgical resection were younger and more likely to be female, to live in an urban area, to have an annual income $>\$ 35,000$, and to undergo treatment at an academic facility. The majority of surgical patients underwent anatomic resection with either lobectomy $(79 \%)$ or pneumonectomy $(15 \%)$. Final pathological analysis showed pN0 status in $18 \%, \mathrm{pN} 1$ in $63 \%$, and $\mathrm{pN} 2 / \mathrm{N} 3$ in $11 \%$. The mean hospital length of stay for surgical patients was $7.7 \pm 8.7$ days (median, 6.0 days), and 30-day mortality in the surgical cohort was $3.5 \%$.

Multivariate logistic regression modeling identified several variables associated with surgical resection, including treatment at an academic facility, Caucasian race, and a higher annual income (Table 3). Increasing age and T2 status were associated with nonoperative management. Unadjusted median OS was greater in those undergoing surgery than in those receiving CRT $(41.5$ months $[95 \% \mathrm{CI}$, 39.8-43.2 months] vs 21.4 months [95\% CI, 20.522.4 months]; $P<.001$ ) (Figure 2 and Table E3).

Adjusted survival analysis was performed using propensity matching based on criteria described in Methods. Propensity score matching identified 2308 patient pairs undergoing either surgical resection or definitive CRT. Results of propensity score matching are shown in Table 4. In this matched-pair analysis, surgical resection was associated with longer median OS compared with definitive 


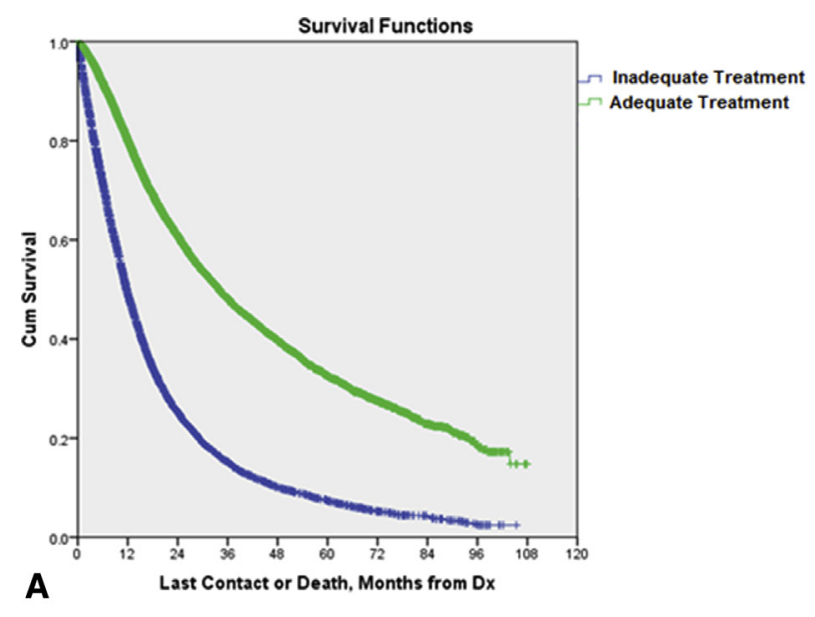

$\underset{0}{0}$

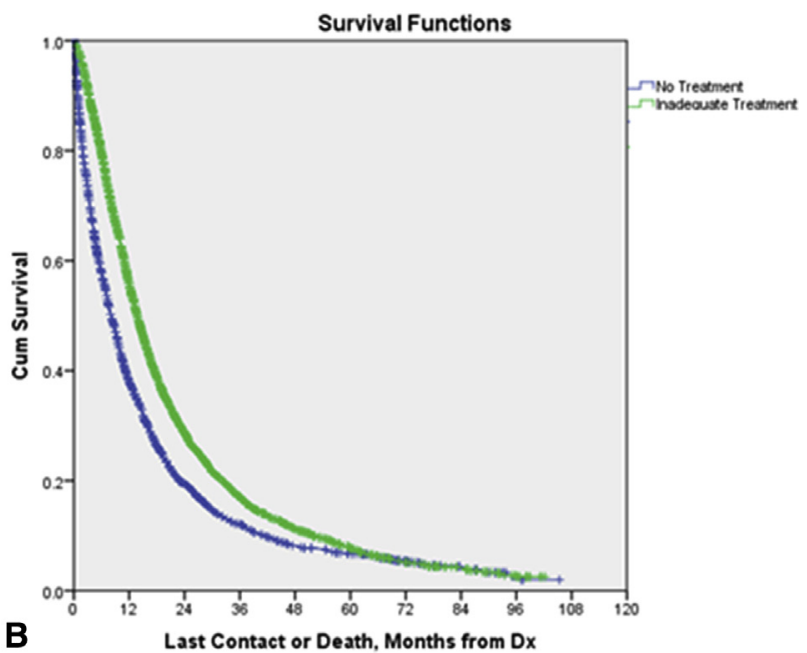

FIGURE 1. Kaplan-Meier curves for OS in patients with clinical N1-positive NSCLC receiving (A) adequate treatment versus inadequate or no treatment and (B) inadequate treatment versus no treatment $(P<$ .001 for both). Cum, Cumulative; Dx, diagnosis. (See Tables E1 and E2 for $95 \%$ confidence intervals and number at risk periodically with time.)

CRT (median OS, 34.1 months [95\% CI, 31.8-36.3 months] versus 22.0 months [95\% CI, 20.8-23.2 months]; $P<.001$ ) (Figure 3 and Table E4).

To assess the impact of adjuvant chemotherapy, we performed an additional survival analysis in surgically resected patients found to be pathological N1 or higher. More than one-half $(53 \%)$ of these resected pathological node-positive patients received adjuvant chemotherapy. Adjuvant treatment in these patients was associated with significantly longer OS (median OS, 48.2 months [95\% CI, 45.0-51.4 months] vs 31.7 months $[95 \%$ CI, 29.1-34.3 months]; $P<.001$ ).

In a parallel analysis, we studied NCDB patients who had undergone complete resection for pathological T1-2N1 disease $(n=7317)$ and received adjuvant treatment. Of
TABLE 2. Demographic and clinical information for patients with N1positive NSCLC undergoing surgical resection and those undergoing adequate definitive CRT

\begin{tabular}{|c|c|c|c|}
\hline Patient characteristic & $\begin{array}{c}\text { Definitive } \\
\text { CRT } \\
(\mathbf{n}=\mathbf{3 1 3 8})\end{array}$ & $\begin{array}{c}\text { Surgical } \\
\text { resection } \\
(\mathbf{n}=\mathbf{9 7 1 9})\end{array}$ & $\begin{array}{c}P \\
\text { value }\end{array}$ \\
\hline Age at diagnosis, $y$, mean $\pm S D$ & $69.4 \pm 9.5$ & $66.0 \pm 10.1$ & $<.001$ \\
\hline Male sex, & $1793(57)$ & $5306(55)$ & .01 \\
\hline Caucasian race, & $2741(87)$ & $8587(88)$ & .13 \\
\hline Academic center & $645(21)$ & $3283(34)$ & $<.001$ \\
\hline Annual income $>\$ 35,000$ & $1811(60)$ & $6069(66)$ & $<.001$ \\
\hline Urban population area & $1844(59)$ & $6111(63)$ & $<.001$ \\
\hline \multicolumn{4}{|l|}{ Charlson/Deyo score } \\
\hline 0 & $1864(59)$ & $5411(56)$ & \multirow[t]{3}{*}{$<.001$} \\
\hline 1 & $895(28)$ & $3226(33)$ & \\
\hline 2 & $379(12)$ & $1082(11)$ & \\
\hline Tumor size, mm, mean \pm SD & $39.9 \pm 28.1$ & $39.8 \pm 36.8$ & .84 \\
\hline \multicolumn{4}{|l|}{ AJCC clinical $\mathrm{T}$ stage, $\mathrm{n}(\%)$} \\
\hline 1 & $937(30)$ & $3692(38)$ & \multirow[t]{2}{*}{$<.001$} \\
\hline 2 & $2201(70)$ & $6027(62)$ & \\
\hline \multicolumn{4}{|l|}{ AJCC clinical stage group, $\mathrm{n}(\%)$} \\
\hline $2 \mathrm{~A}$ & $1090(35)$ & $4232(43)$ & \multirow[t]{3}{*}{$<.001$} \\
\hline $2 \mathrm{~B}$ & $1963(63)$ & $5180(53)$ & \\
\hline 2 NOS & $85(3)$ & $307(3)$ & \\
\hline \multicolumn{4}{|l|}{ Type of resection, $n(\%)$} \\
\hline None & $3138(100)$ & 0 & \multirow[t]{4}{*}{$<.001$} \\
\hline Wedge & 0 & $612(6)$ & \\
\hline Lobectomy & 0 & 7657 (79) & \\
\hline Pneumonectomy & 0 & $1450(15)$ & \\
\hline \multicolumn{4}{|l|}{ AJCC pathological T stage, $\mathrm{n}(\%)$} \\
\hline 0/IS & NA & $47(<1)$ & \multirow[t]{6}{*}{ NA } \\
\hline 1 & NA & $2922(30)$ & \\
\hline 2 & NA & $5279(54)$ & \\
\hline 3 & NA & $398(4)$ & \\
\hline 4 & NA & $298(3)$ & \\
\hline $\mathrm{X}$ & NA & $775(8)$ & \\
\hline \multicolumn{4}{|l|}{ AJCC pathological N stage, $\mathrm{n}(\%)$} \\
\hline 0 & NA & $1758(18)$ & \multirow[t]{5}{*}{ NA } \\
\hline 1 & NA & $6127(63)$ & \\
\hline 2 & NA & $1006(10$ & \\
\hline 3 & NA & $17(<1)$ & \\
\hline $\mathrm{X}$ & NA & $811(8)$ & \\
\hline Any radiotherapy & $3138(100)$ & $1772(18)$ & $<.001$ \\
\hline $\begin{array}{l}\text { Total radiation dose, cGy, } \\
\text { mean } \pm \mathrm{SD}\end{array}$ & $6669 \pm 3695$ & $5423 \pm 2533$ & $<.001$ \\
\hline \multicolumn{4}{|l|}{ Chemotherapy } \\
\hline None & NA & $4613(47)$ & \multirow[t]{4}{*}{$<.001$} \\
\hline Single agent & $320(10)$ & $239(2)$ & \\
\hline Multiagent & $2509(80)$ & $4328(45)$ & \\
\hline Chemotherapy NOS & $309(10)$ & $539(6)$ & \\
\hline
\end{tabular}

$C R T$, Chemoradiation; $S D$, standard deviation; $A J C C$, American Joint Committee on Cancer; NOS, not otherwise specified; $I S$, in situ; $N A$, not applicable.

these patients, $6438(88 \%)$ received adjuvant chemotherapy and the other $879(12 \%)$ received adjuvant CRT. The sole factor associated with the administration of radiation therapy in addition to chemotherapy in the adjuvant setting was pathological T2 status (OR, 1.55; 
TABLE 3. Multivariable logistic regression analysis identifying variables associated with surgical resection in clinical N1 nodepositive NSCLC

\begin{tabular}{lcc}
\hline Patient and treatment variable & OR $(\mathbf{9 5} \% \mathbf{C I})$ & $\boldsymbol{P}$ value \\
\hline Age & $0.96(0.96-0.97)$ & $<.001$ \\
Academic facility & $1.91(1.71-2.12)$ & $<.001$ \\
Caucasian race & $1.34(1.17-1.54)$ & $<.001$ \\
Annual income $>\$ 35,000$ & $1.22(1.10-1.35)$ & $<.001$ \\
Urban area & $1.10(0.99-1.22)$ & .05 \\
Charlson score 1 & $1.27(1.15-1.40)$ & $<.001$ \\
Charlson score 2 & $1.06(0.92-1.22)$ & .42 \\
Clinical T2 status & $0.72(0.65-0.80)$ & $<.001$ \\
\hline OR, Odd
\end{tabular}

$O R$, Odds ratio; $C I$, confidence interval.

95\% CI, 1.29-1.85). Factors associated with chemotherapy alone were treatment at an academic center (OR, 0.50; 95\% CI, 0.42-0.61) and lobectomy or pneumonectomy (OR, 0.39 [95\% CI, 0.29-0.52] and 0.24 [95\% CI, 0.16-0.36], respectively, compared with sublobar resection). In a multivariable Cox proportional hazards model, lobectomy and pneumonectomy were associated with prolonged survival in this group (hazard ratio [HR], 0.65 [95\% CI, 0.54-0.79] and 0.74 [95\% CI, 0.59-0.93], respectively, compared with sublobar resection), whereas factors associated with shorter OS included age (HR, 1.02; 95\% CI, 1.01-1.02), male sex (HR, 1.29; 95\% CI, 1.18-1.42), Caucasian race (HR, 1.24; 95\% CI, 1.06-1.45), pT2 status (HR, 1.15; 95\% CI, 1.03-1.28), and treatment with CRT versus chemotherapy alone (HR, 1.56; $95 \%$ CI,1.38-1.77).

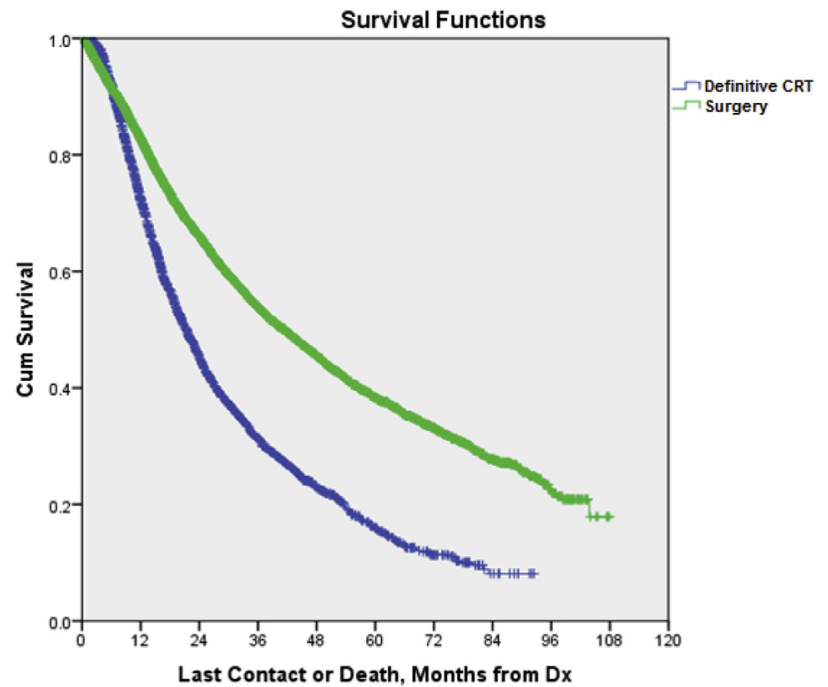

FIGURE 2. Kaplan-Meier curve for OS in patients with clinical N1-positive NSCLC undergoing definitive CRT versus those undergoing surgical resection $(P<.001)$. Cum, Cumulative; $D x$, diagnosis; $C R T$, chemoradiation. (See Table E3 for $95 \%$ confidence intervals and number at risk periodically with time.)
TABLE 4. Results of propensity score matching of 2308 patient pairs undergoing either surgical resection or definitive CRT

\begin{tabular}{|c|c|c|c|}
\hline Patient characteristic & $\begin{array}{c}\text { Definitive } \\
\text { CRT } \\
(\mathbf{n}=\mathbf{2 3 0 8})\end{array}$ & $\begin{array}{c}\text { Surgical } \\
\text { resection } \\
(\mathbf{n}=\mathbf{2 3 0 8})\end{array}$ & $\begin{array}{c}P \\
\text { value }\end{array}$ \\
\hline Age at diagnosis, $\mathrm{y}$, mean $\pm \mathrm{SD}$ & $68.9 \pm 9.0$ & $68.9 \pm 8.9$ & .81 \\
\hline Male sex, $\mathrm{n}(\%)$ & $1279(55)$ & $1292(56)$ & .72 \\
\hline Caucasian race, $\mathrm{n}(\%)$ & $2030(88)$ & $2024(88)$ & .82 \\
\hline Academic center, n $(\%)$ & $508(22)$ & $511(22)$ & .94 \\
\hline Annual income $>\$ 35,000, \mathrm{n}(\%)$ & $1450(63)$ & $1415(61)$ & .30 \\
\hline \multicolumn{4}{|l|}{ Charlson/Deyo score, n (\%) } \\
\hline 0 & $1317(57)$ & $1304(56)$ & .91 \\
\hline 1 & $710(31)$ & $715(31)$ & \\
\hline 2 & $281(12)$ & $289(13)$ & \\
\hline Tumor size, $\mathrm{mm}$, mean $\pm \mathrm{SD}$ & $39.8 \pm 28.6$ & $39.7 \pm 34.2$ & .90 \\
\hline Radiotherapy & $2308(100)$ & $397(17)$ & $<.001$ \\
\hline $\begin{array}{l}\text { Total radiation dose, cGy, } \\
\text { mean } \pm \mathrm{SD}\end{array}$ & $6619 \pm 3409$ & $5293 \pm 1580$ & $<.001$ \\
\hline \multicolumn{4}{|l|}{ Chemotherapy } \\
\hline None & $0(0)$ & $1172(51)$ & $<.001$ \\
\hline Single agent & $230(10)$ & $50(2)$ & \\
\hline Multiagent & $1862(81)$ & $954(41)$ & \\
\hline Chemotherapy NOS & $216(9)$ & $132(6)$ & \\
\hline
\end{tabular}

Patients were matched for variables including age, race, sex, income, type of treatment facility, comorbidity score, and tumor size. CRT, Chemoradiation; $S D$, standard deviation; NOS, not otherwise specified.

\section{DISCUSSION}

This analysis of data from the NCDB brings to light several interesting points regarding the treatment of clinical N1 node-positive NSCLC in the United States. First, despite evidence showing significant long-term survival with surgical resection and clear guidelines recommending its

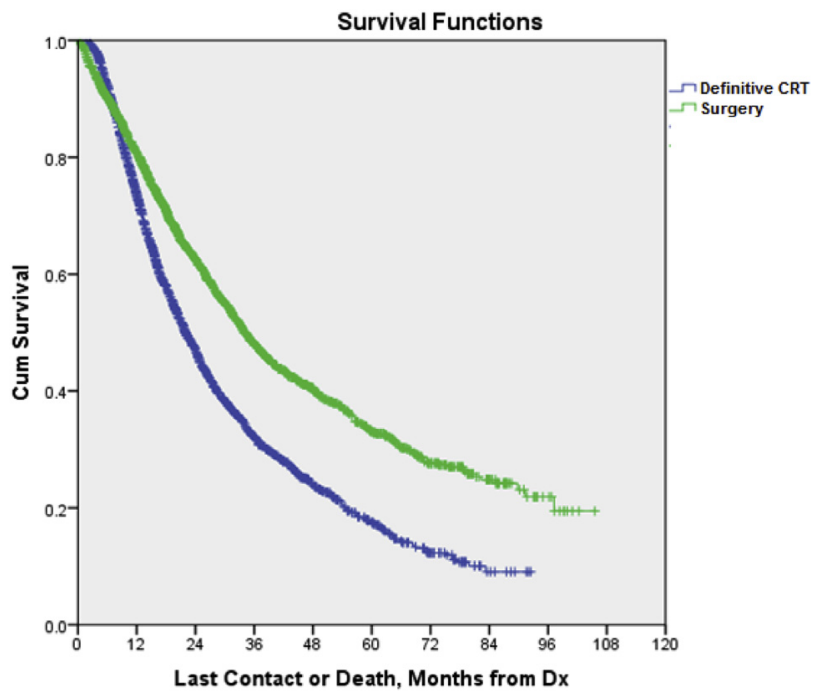

FIGURE 3. Kaplan-Meier curve for OS in 2308 propensity scorematched patient pairs undergoing either surgical resection or definitive CRT for the treatment of N1 node-positive NSCLC $(P<.001)$. Cum, Cumulative; $D x$, diagnosis; CRT, chemoradiation. (See Table E4 for $95 \%$ confidence intervals and number at risk periodically with time.) 
use in this population, less than one-half $(48 \%)$ of patients presenting with clinical T1-2/N1 disease underwent surgical resection as part of their treatment. ${ }^{2-4}$ Our propensity-matched analysis confirms a longer OS in patients selected for surgical resection compared with those treated with adequate definitive CRT (median OS, 34.1 months vs 22.0 months; $P<.001$ ). Although the reason for the discrepancy between the guidelines and nationwide practices is likely multifactorial, our regression analysis identified several variables significantly associated with resection including younger age and smaller tumors. In addition, our multivariate analysis revealed that demographic and socioeconomic factors, such as race and income, play roles as well.

Interestingly, receipt of treatment at an academic center was associated with a greater likelihood of undergoing surgical resection. Although there is some evidence indicating that treatment in an academic institution may be associated with improved outcomes after surgery for lung cancer, the use of surgical resection itself has not previously served as a quality measure. ${ }^{11}$ Because academic centers tend to be associated with higher volume and greater surgeon experience, possible explanations for this relationship between academic institutions and the use of surgical resection may include stricter adherence to published guidelines, greater willingness to attempt resection in cases in which it might not be technically feasible, and a higher threshold for declaring patients medically inoperable.

Regardless, even in light of these factors, the relatively low incidence of surgical resection in this patient population is surprising. Perhaps the most straightforward assumption would be that patients are not offered resection owing to medical or technical inoperability. However, it is interesting to note that of adequately treated patients, the percentage with a Charlson/Deyo comorbidity score of 1 or $\geq 2$ was higher in the surgical resection group compared with the CRT group ( $44 \%$ vs $41 \% ; P<.001$ ). Even patients who received inadequate or no treatment had a lower percentage with a Charlson/Deyo score $\geq 1$ than patients who underwent surgery ( $41 \%$ vs $42 \%)$. Thus, there was no clear evidence from the NCDB data indicating that these patients were denied surgery based on medical comorbidity. Nonetheless, it is important to keep in mind that the true extent of a patient's operability extends beyond the Charlson/Deyo score. Unfortunately, the NCDB does not capture more detailed information on pulmonary function, such as preoperative forced expiratory volume in 1 second or diffusion capacity for carbon monoxide.

Assuming that all patients failing to undergo resection are truly medically inoperable, nonoperative treatment of this population falls short of established guidelines. Although it must be noted that randomized trials specifically evaluating medically inoperable patients with N1-node positive NSCLC are lacking, extrapolation of data from patients with stage IIIA and IIIB disease supports combined CRT as the standard of care. ${ }^{4-7}$ In light of this, our data reveal that only $29 \%$ of the 10,647 patients with T1-2N1 NSCLC not undergoing resection received what we had defined as adequate CRT (ie, chemotherapy in conjunction with $>4500$ cGy of radiation in any order).

In patients who received some form of therapy but failed to meet the standard of adequate treatment, the major barrier appeared to be chemotherapy. The majority $(72 \%)$ of these inadequately treated patients received external beam radiation (mean radiation dose, $5334 \mathrm{cGy}$ ), whereas more than one-half $(52 \%)$ did not receive any chemotherapy and another $6 \%$ received only single-agent treatment.

Although several large trials have shown adjuvant chemotherapy to be beneficial in patients with completely resected stage II NSCLC, our data suggest that administering chemotherapy appears to be difficult in the adjuvant setting. ${ }^{12-14}$ According to the NCDB data, only $53 \%$ of pathological N1 node-positive patients undergoing resection received perioperative chemotherapy. Once again, this suggests a lack of adherence to accepted guidelines stating that surgical resection alone is not a recommended therapy for this patient population. ${ }^{3}$

Perhaps one of the most encouraging aspects of this analysis is that the vast majority of patients undergoing surgery received an anatomic resection. According to the NCDB, of those with clinical T1-2N1 NSCLC, 79\% underwent lobectomy, $15 \%$ received pneumonectomy, and only $6 \%$ were treated with wedge resection. Thus, once patients proceeded to surgery, their treatment appeared to be in line with current standards of care regarding operative intervention. ${ }^{15,16}$

The use of adjuvant radiation following complete resection of NSCLC is controversial. Several studies suggest lack of benefit and even diminished survival in patients with early-stage disease. ${ }^{17-19}$ However, some centers still include adjuvant radiotherapy in treatment protocols for this patient population. Our data from the NCDB support the notion that adjuvant radiation in completely resected patients with T1-2N1 NSCLC is associated with shorter OS compared with treatment with adjuvant chemotherapy alone. Unless additional studies provide strong evidence for a benefit in this population, routine treatment with radiotherapy should be avoided in these patients.

One additional factor that warrants discussion is the discrepancy between clinical and pathological staging in a substantial proportion of cases. Several previous studies have documented a poor correlation between clinical and pathological staging in patients with NSCLC. ${ }^{20-22}$ In 2011, Darling and colleagues ${ }^{22}$ evaluated 149 patients undergoing both positron emission tomography (PET)/computed tomography (CT) and invasive mediastinal staging (mediastinoscopy, thoracotomy, or both) to evaluate the accuracy of the preoperative staging workup, 
and found a fairly poor correlation between the 2 methods (64\% positive predictive value, $95 \%$ negative predictive value). Of the 13 patients with clinical N1 disease on PETCT, $1(8 \%)$ was pathological node-negative and $4(31 \%)$ had $\mathrm{N} 2$ disease on final pathology. Our study confirms these findings in a much larger cohort of patients. According to the NDCB, of patients with clinical N1 node-positive NSCLC, $18 \%$ showed no evidence of nodal disease on final pathological evaluation. It is possible that node-positivity on clinical staging evaluation biases providers against referral for surgical resection. If so, these data suggest that false-positive clinical staging evaluations may prevent resection in a substantial proportion of patients with true pathological stage I disease. Conversely, although the study excluded patients with clinical evidence of mediastinal lymph node involvement, the incidence of pathologic $\mathrm{N} 2$ or N3 disease was $>10 \%$ in resected patients. These data once again highlight the relative inadequacy of current clinical staging paradigms.

The discrepancy between published standards of care and the reality of actual physician practice highlighted in this study underscores the need for additional measures to improve the treatment of patients with N1 node-positive NSCLC across the country. Demographic and clinical factors, such as patient age and tumor size, are fairly immutable; however, the impact of socioeconomic variables suggests that efforts to improve access to healthcare in disadvantaged populations could potentially yield benefits by making surgery available to a greater proportion of operable patients. It is possible that popularization of healthcare-based Internet portals and mobile device applications may allow for improved delivery of care to these underserved populations.

Although a certain subset of patients will undoubtedly be inoperable due to medical comorbidity, it is hard to believe that this subset would constitute more than one-half of the patients identified in the database. This finding prompts us to speculate that these patients are not referred for surgical resection owing to reluctance on behalf of either primary physicians or the patients themselves. Multidisciplinary evaluation of these patients should be mandatory, and improved communication between surgeons and referring physicians may eliminate preconceived misconceptions regarding the safety and efficacy of surgery in this population. Similarly, enhanced dialogue between surgeons and their patients can help to alleviate additional barriers to operative intervention, such as anxiety regarding postoperative pain and lack of perceived benefit.

This study has several limitations. Although the NCDB contains a wealth of patient data, the information is still reviewed retrospectively, and treatment groups are not randomized. Even with the application of such techniques as multivariate analysis and propensity score matching, it is possible that more subtle difference in patient populations exist and thus bias results. Similarly, several relevant patient variables are not captured by the database. As a result, factors such as staging modalities and preoperative forced expiratory volume or other measures of operative fitness cannot be compared in the current analysis. Although the majority of patients likely underwent clinical staging with CT and/or PET, the use of these modalities is not mandated, nor is it recorded by the NCDB.

In addition, this analysis includes only patients treated before 2010. It is possible that practice patterns have changed since that time, as the prevalence of minimally invasive surgical modalities has increased and the database supporting adjuvant therapy has grown. Finally, although the American College of Surgeons Commission on Cancer requires at least a $90 \%$ patient follow-up rate as part of its accreditation program, our deidentified patient information cannot be used to further validate the accuracy of survival information submitted to the NCDB.

Our review of the NCDB reveals that current treatment of patients with N1-node positive NSCLC falls short of established guidelines. Although data from the NCDB confirm a longer OS associated with surgery, a large number of patients fail to undergo resection. In addition, medical therapy of patients not selected for surgery is suboptimal in a large percentage of cases. More research is needed to elucidate the reasons behind these delinquencies and improve patient care in the future. The impact of such factors as socioeconomic status and treatment at an academic center highlight the need for improved access to care in certain patient populations. In addition, these data highlight the importance of multidisciplinary evaluation in this patient population. Perhaps better communication between surgeons and referring physicians, as well as better dialogue between surgeons and their patients, may help alleviate barriers to surgical resection in operable patients.

\section{Conflict of Interest Statement}

Daniel Morgensztern reports speaker fees for BoehringerIngelheim and Genentech, and advisory board work for Genentech, Heat Biologics, and Celgene. Clifford Robinson reports Research Support from and work on the speaker's bureau for Varian Medical Systems, as well as consulting work for and stock ownership in Radialogica. All other authors have nothing to disclose with regard to commercial support.

\section{References}

1. Detterbeck FC, Gibson CJ. Turning gray: the natural history of lung cancer over time. J Thorac Oncol. 2008;3:781-92.

2. Chansky K, Sculier JP, Crowley JJ, Giroux D, Van Meerbeeck J, Goldstraw P. The International Association for the Study of Lung Cancer Staging Project: prognostic factors and pathologic TNM stage in surgically managed non-small cell lung cancer. J Thorac Oncol. 2009;4:792-801.

3. Howington JA, Blum MG, Chang AC, Balekian AA, Murthy SC. Treatment of stage I and II non-small cell lung cancer: diagnosis and management of lung cancer, 3rd ed: American College of Chest Physicians evidence-based clinical practice guidelines. Chest. 2013;143(5 Suppl):e278S-313. 
4. National Comprehensive Cancer Network. NCCN guidelines, version 5. 2015: non-small cell lung cancer. Available at: http://www.nccn.org/professionals/ physician_gls/pdf/nscl.pdf. Accessed October 20, 2015.

5. Aupérin A, Le Péchoux C, Rolland E, Curran WJ, Furuse K, Fournel P, et al. Meta-analysis of concomitant versus sequential radiochemotherapy in locally advanced non-small-cell lung cancer. J Clin Oncol. 2010;28:2181-90.

6. O'Rourke N, Roqué I, Figuls MR, Farré Bernadó N, Macbeth F. Concurrent chemoradiotherapy in non-small cell lung cancer. Cochrane Database Syst Rev. 2010;6:CD002140.

7. Curran WJ Jr, Paulus R, Langer CJ, Komaki R, Lee JS, Hauser S, et al. Sequential vs. concurrent chemoradiation for stage III non-small cell lung cancer: randomized phase III trial RTOG 9410. J Natl Cancer Inst. 2011;103:1452-60.

8. Bilimoria KY, Stewart AK, Winchester DP, Ko CY. The National Cancer Data Base: a powerful initiative to improve cancer care in the United States. Ann Surg Oncol. 2008;15:683-90.

9. Rusch VW, Asamura H, Watanabe H, Giroux DJ, Rami-Porta R, Goldstraw P. The IASLC lung cancer staging project: a proposal for a new international lymph node map in the forthcoming seventh edition of the TNM classification of lung cancer. J Thorac Oncol. 2009;4:568-77.

10. Edge S, Byrd DR, Compton CC, Fritz AG, Greene FL, Trotti A, eds. AJCC Cancer Staging Manual. 7th ed. New York: Springer; 2010.

11. Meguid RA, Brooke BS, Chang DC, Sherwood JT, Brock MV, Yang SC. Are surgical outcomes for lung cancer resections improved at teaching hospitals? Ann Thorac Surg. 2008;85:1015-24.

12. Winton T, Livingston R, Johnson D, Rigas J, Johnston M, Butts C, et al. Vinorelbine plus cisplatin vs observation in resected non-small-cell lung cancer. N Engl J Med. 2005;352:2589-97.

13. Douillard JY, Rosell R, De Lena M, Carpagnano F, Ramlau R, GonzálesLarriba JL, et al. Adjuvant vinorelbine plus cisplatin versus observation in patients with completely resected stage IB-IIIA non-small-cell lung cancer (Adjuvant Navelbine International Trialist Association [ANITA]): a randomized controlled trial. Lancet Oncol. 2006;7:719-27.

14. Pignon JP, Tribodet H, Scagliotti G, Douillard JY, Shepherd FA, Stephens RJ, et al. Lung adjuvant cisplatin evaluation: a pooled analysis by the LACE Collaborative Group. J Clin Oncol. 2008;26:3552-9.

15. Ginsberg RJ, Rubinstein LV. Randomized trial of lobectomy versus limited resection for T1 N0 non-small cell lung cancer. Lung Cancer Study Group. Ann Thorac Surg. 1995;60:615-22.

16. Miller DL, Rowland CM, Deschamps C, Allen MS, Trastek VF, Pairolero PC Surgical treatment of non-small cell lung cancer $1 \mathrm{~cm}$ or less in diameter. Ann Thorac Surg. 2002;73:1545-50.

17. Burdett S, Rydzewska L, Tierney JF, Fisher DJ. A closer look at the effects of postoperative radiotherapy by stage and nodal status: updated results of an individual participant data meta-analysis in non-small-cell lung cancer. Lung Cancer. 2013;80:350-2

18. Feng QF, Wang M, Wang LJ, Yang ZY, Zhang YG, Zhang DW, et al. A study of postoperative radiotherapy in patients with non-small-cell lung cancer: a randomized trial. Int J Radiat Oncol Biol Phys. 2000;47:925-9.

19. Dautzenberg B, Arriagada R, Chammard AB, Jarema A, Mezzetti M, Mattson K, et al. A controlled study of postoperative radiotherapy for patients with completely resected nonsmall cell lung carcinoma. Group d'Etude et de Traitement des Cancers Bronchiques. Cancer. 1999;86:265-73.

20. D'Cunha J, Corfits AL, Herndon JE II, Kern JA, Kohman LJ, Patterson GA, et al. Molecular staging of lung cancer: real-time polymerase chain reaction estimation of lymph node micrometastatic tumor cell burden in stage I non-small cell lung cancer. Preliminary results of Cancer and Leukemia Group B Trial 9761. J Thorac Cardiovasc Surg. 2002;123:484-91.

21. López-Encuentra A, Garcia-Luján R, Rivas JJ, Rodriguez-Rodriguez J, TorresLanza J, Varela-Simo G. Comparison between clinical and pathologic staging in 2,994 cases of lung cancer. Ann Thorac Surg. 2005;79:974-9.

22. Darling GE, Maziak DE, Inculet RI, Gulenchyn KY, Driedger AA, Ung YC, et al. Positron emission tomography-computed tomography compared with invasive mediastinal staging in non-small cell lung cancer: results of mediastinal staging in the Early Lung Positron Emission Tomography Trial. J Thorac Oncol. 2011;6:1367-72.

Key Words: non-small cell lung cancer, locally advanced, lymph nodes, surgical resection, multi-modality therapy, practice patterns

\section{Discussion}

Dr Bott (St Louis, Mo). Good morning. On behalf of my coauthors, I would like to thank the association for the opportunity to present our study entitled "Patterns of care in hilar node-positive non-small cell lung cancer: A missed treatment opportunity." We have no relevant disclosures.

Non-small cell lung cancer metastatic to regional lymph nodes is an aggressive disease with high mortality. However, in patients with disease limited to pulmonary and hilar nodes, long-term survival is possible with multimodality therapy. For patients with resectable tumors, and metastases limited to these N1 nodes, surgical resection forms a foundation of therapy. For patients unfit to tolerate surgery, randomized data are lacking; however, national guidelines extrapolating data from studies of $\mathrm{N} 2$ node-positive patients consider definitive chemoradiation a preferred standard of care. Despite the publication of these guidelines from such groups as the National Comprehensive Cancer Network and the American College of Chest Physicians, adherence to these protocols at a national level is unknown. To investigate this, we used the National Cancer Database (NCDB). The NCDB was created in 1989 as a joint effort on behalf of the Commission on Cancer, the American College of Surgeons, and the American Cancer Society. All members of the Commission on Cancer are required to submit comprehensive demographic, clinical, and outcomes information to the database. It is estimated that the NCDB captures approximately $70 \%$ of all cancer cases diagnosed in the United States annually. To better characterize the treatment of $\mathrm{N} 1$ node- positive non-small cell lung cancer in the United States, we queried the NCDB to identify patients treated for clinical N1 node-positive disease between 1998 and 2010. We limited our study to patients with clinical T1 or T2 tumors, corresponding to those with stage II disease based on the most recent AJCC staging system. Those patients with clinically positive mediastinal lymph nodes were specifically excluded. We considered patients undergoing surgical resection or chemotherapy with at least 45 Gy of radiation as adequately treated, and considered patients undergoing either isolated chemotherapy or radiation or receiving less than 45 Gy of radiation as part of combination chemotherapy as inadequately treated. Our search identified 20,366 patients who met the study criteria. Of these, only $63 \%$ received what we had defined as adequate therapy. Of all patients with stage II disease, only 48\% - less than one-half-underwent surgical resection, and an additional $15 \%$ received adequate definitive chemoradiation. Approximately $25 \%$ of patients received inadequate chemoradiation, and $14 \%$ received no treatment.

Univariate analysis showed that patients treated with adequate therapy were younger, were more likely to be treated at an academic center, and had a higher annual income, lower comorbidity score, and smaller tumors both in terms of lower $\mathrm{T}$ 
stage and a higher incidence of stage IIa versus IIb disease. Kaplan-Meier analysis for overall survival showed that patients treated with adequate therapy had improved overall survival compared with those receiving no or inadequate treatment. Median overall survival was 34 months in adequately treated patients versus 11.7 months in those receiving no therapy or inadequate therapy. Similar analysis showed a less impressive yet still significant survival benefit for patients receiving inadequate chemoradiation compared with those receiving no therapy. Of the 9719 patients undergoing surgery, the vast majority received an anatomic resection in the form of lobectomy or pneumonectomy. The results of pathological staging in these patients indicate that $63 \%$ of clinical N1 patients were pathological N1, whereas almost $20 \%$ were pathological node-negative and $11 \%$ were pathological N2 or higher. Approximately $50 \%$ of the resected patients received chemotherapy, and $18 \%$ received some form of postoperative radiation. Univariate analysis of the patients undergoing surgical resection versus those receiving adequate definitive chemoradiation showed that the surgical patients were more likely to be younger, to be female, to undergo treatment at an academic center, to have a higher income, and to reside in an urban area. The surgical patients also had slightly lower comorbidity scores and were more likely to be clinical T1 versus T2. Many of these differences were confirmed in a multivariate model. Here younger age, treatment in an academic facility, Caucasian race, higher income, and T1 versus T2 status were all associated with increased likelihood of surgical resection. Kaplan-Meier analysis for overall survival showed that the patients selected for surgery had improved survival compared with those undergoing definitive chemoradiation. Median overall survival was 41.5 months in the surgical group, compared with 21.4 months in the chemoradiation group.

To account for the inherent differences in the operative and nonoperative patient populations, we performed propensity score matching to more clearly define the survival benefit associated with surgical resection. Propensity score matching for the variable shown here resulted in 2308 matched patient pairs. Propensity-matched analysis still showed increased survival in the resected patients, with a median overall survival of 34 months, compared with 22 months in the patients receiving definitive chemoradiation.

There are some limitations to this analysis. First, although the NCDB is a robust resource with data on a large number of patients, the data are still reviewed retrospectively, which does not take the place of a randomized clinical trial. In addition, although the database does record gross assessments and functional status such as the Charlson/Deyo score, more detailed measures of operability, such as preoperative pulmonary function tests, are lacking. In addition, although most patients likely undergo staging using current PET/CT-based protocols, exact information regarding the staging workup is not recorded.
In conclusion, our analysis of the National Cancer Data Base suggests that the treatment of patients with N1-positive non-small cell lung cancer falls far short of established guidelines in a substantial percentage of cases. Despite confirming a survival benefit for surgery, less than one-half the patients identified in the NCDB underwent resection. In addition, patients selected for chemoradiation were undertreated in a high percentage of cases. The impact of demographic and socioeconomic factors on surgical resection highlights the need for improved access to care in certain patient populations. In light of this information, we recommend that patients with clinical N1 non-small cell lung cancer be evaluated in a multidisciplinary setting, and that surgical evaluation of these patients be mandatory. On the part of surgeons, better communication with referring physicians and improved dialogue with patients may alleviate barriers to resection in operable patients. Thank you for your attention.

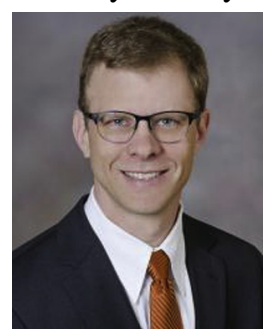

Dr P. Schipper (Portland, Ore). So, Dr Bott, nice study, nicely presented. You essentially made 2 comparisons, one was this propensity analysis, looking at adequately treated patients and chemoradiation alone versus with the addition of surgery, and other than the fact that it is a retrospective data-based study. I can't find fault with that part of your paper. I can't rap it in any way, and I think it seems to be a valid conclusion. It is interesting that you sort of put it as a subtext to your paper, because I think that is the meat of what you presented to us today.

It is the second comparison that I'm not quite convinced about, and I had a couple of questions, and this is the inadequately versus adequately treated patients with N1 disease. So N1 disease is a little tricky. It's tricky to clinically stage, as you have shown, and I don't think you actually put that data up there, but in the paper you talked about the fact that one-third of the patients, roughly, who had clinical N1 disease actually did not end up having that in the pathological diagnosis, and in fact for every 1 case that was upstaged, 2 cases were down-staged, so apparently despite fairly clear written instructions from the NCCN, the treatment of $\mathrm{N} 1$ disease is also complicated.

So to kind of better understand this database, I have 2 questions for you. The first question is, just to know, these 20,000 patients, what percentage of what is in this database during this time period does that represent? Because $\mathrm{N} 1$ is a little tricky to get into a data base to currently clinically stage, if that is $2 \%$ of the lung cancer patients in the database, it might be a little suspect that they are putting people in the right categories. Alternatively, if it's $25 \%$ of the patients in the database, then you also might suspect that they have some people incorrectly clinically staged. 
Do you have any numbers of which patients were in other stage categories?

Dr Bott. Sure, so during the same time period, 1998 to 2010, there were about 1 million patients with non-small cell lung cancer in the database with clinical staging information available. The bulk of those patients, almost half, are stage IV patients actually, so the lower-stage groups get whittled down a little bit, and of those lower-stage groups, the majority are actually stage I and stage III. The stage II patients really only make up about 50,000 patients or so within that million, so it's a fairly small subset. I think that speaks to, as you were saying, the difficulty of diagnosing stage II disease preoperatively, so I suspect that that's sort of the causality there, but I agree that it is a smaller percentage of the overall patient population than maybe some of the other stage groupings.

Dr Schipper. The second question and last question. I would like you to consider another variable, and I'm going to call that variable "best possible treatment." Maybe not adequate according to the NCCN guidelines, but the best treatment that a multidisciplinary group looking at a patient with comorbidities could do where they are not increasing the harm or increasing the mortality just because of the treatment, and you have worked with this database, and you have seen the variables that it gives you. You do not get to choose those variables. If you were to come up with what you want to look at to determine if the best possible treatment is being delivered to these folks with N1 disease, what things would you look for to make that decision?

Dr Bott. Sure, so I think that the real critical situation is to determine what percentage of those patients are truly operable, and that's a difficult thing to do. I spoke to it a little bit in terms of the preoperative pulmonary function. I suspect that's where a large percentage of those patients are lost in terms of their resectability. So I think that's important. The Charlson score which is included in the database is sort of a gross measure, but does not really capture maybe more, sort of subtle nuances in operability, so I think the pulmonary function tests would be the main one. I think there's a subjective sort of aspect to this that is difficult to quantify at any database, but I'm not sure that's really going to be assessable regardless of what the source of the data is, but at least the pulmonary function would be captured in maybe an institutional data base or something like that and could be drilled down a little bit further.

Dr Schipper. Thank you. Nice work.

Dr D. Wood (Seattle, Wash). A great and very important paper. I have a couple of comments and one questionable question. One thing I think to continue to put as a potential limitation is, although you're able to identify patients who did not have compliant care, unfortunately you can't assess the reasons for noncompliance, that actually may in some cases be patient preference as well, which is another important thing, a variable that you can't measure in a database, and you've approached this very well. I would urge you to be edgier and more definitive. You've found some very important and disturbing findings that warrant being very strong in your concluding statements about it, some are the aspect of cancer disparities. So cancer disparities are a 2015 goal, or eliminating cancer treatment disparities is a 2015 goal of the American Cancer Society. You've demonstrated how there are differences in socioeconomic group, race, age, etc, which are clearly areas that one can emphasize and get some political pressure on the effort to bring compliance to bear.

Paul emphasized another very important point, which is that a third of these patients actually do not even have stage II disease, and that is I think another great opportunity to emphasize how important surgical therapy is, those of course, never get determined when patients get nonsurgical therapy. My question, and I think I know the answer to it, but you did not measure compliance as adjuvant chemotherapy after surgical resection, is that correct?

Dr Bott. We did in the manuscript. It wasn't present, I don't think, in the presentation, but about half of the patients in the surgery group received postoperative chemotherapy. A subset of those are N0, so that's... I don't have information as far as which, of the true N1 patients, which of those received chemotherapy, but...

Dr Wood. That's actually somewhat encouraging, because your time frame of the study spanned when there were no recommendations for adjuvant chemotherapy postsurgery, to the modern era where it is, and so that combined with the pathologic N0 might explain or at least rationalize a significant portion not getting adjuvant chemotherapy, but that's an important part of compliance as well. Nice work.

Unidentified speaker. I had a question and a comment before you leave the podium. A couple of years ago at this meeting, we did a similar analysis looking at neonates with hypoplastic left heart syndrome, and we found that there were very many disparities along some of the same parameters that you've investigated. One of the regrets of that study was that we were not able to look at things like insurance status, and so although you've looked as income as a surrogate for socioeconomic status, I wonder whether in this database you have the ability to do so, because I think it would be a provocative adjunct to what Dr Wood was suggesting, some of the disparities that we seek to eliminate.

Dr Bott. Sure. I don't know that the insurance status is captured in the NCDB per se, but it's something we can certainly try and investigate. 
TABLE E1. Ninety-five percent confidence intervals and number at risk periodically with time for Kaplan-Meier curves (shown in Figure $1, A$ ) for overall survival in patients with clinical N1-positive NSCLC undergoing adequate versus inadequate or no treatment (both $P<.001)$

\begin{tabular}{|c|c|c|c|c|c|c|}
\hline \multirow[b]{2}{*}{ Months } & \multicolumn{3}{|c|}{ Inadequate } & \multicolumn{3}{|c|}{ Adequate } \\
\hline & Mean \pm SD,$\%$ & $95 \% \mathrm{CI}$ & n at risk & Mean \pm SD, $\%$ & $95 \% \mathrm{CI}$ & $\mathrm{n}$ at risk \\
\hline 0 & 100 & & 7509 & 100 & & 12,857 \\
\hline 12 & $49.10 \pm 0.60$ & $47.92-50.28$ & 3287 & $80.10 \pm 0.40$ & $79.32-80.88$ & 9060 \\
\hline 24 & $25.30 \pm 0.50$ & $24.32-26.28$ & 1428 & $60.80 \pm 0.50$ & 59.82-61.78 & 5406 \\
\hline 36 & $15.20 \pm 0.50$ & $14.22-16.18$ & 738 & $48.20 \pm 0.50$ & $47.22-49.18$ & 3229 \\
\hline 48 & $10.20 \pm 0.40$ & $9.42-10.98$ & 417 & $39.70 \pm 0.60$ & $38.52-40.88$ & 1905 \\
\hline 60 & $7.30 \pm 0.40$ & $6.52-8.08$ & 246 & $32.50 \pm 0.60$ & $31.32-33.68$ & 1092 \\
\hline 72 & $5.20 \pm 0.40$ & $4.42-5.98$ & 129 & $27.60 \pm 0.60$ & $26.42-28.78$ & 609 \\
\hline 84 & $4.30 \pm 0.40$ & $3.52-5.08$ & 69 & $22.90 \pm 0.70$ & $21.53-24.27$ & 264 \\
\hline
\end{tabular}

$S D$, Standard deviation; $C I$, confidence interval.

TABLE E2. Ninety-five percent confidence intervals and number at risk periodically with time for Kaplan-Meier curves (shown in Figure $1, B$ ) for overall survival in patients with clinical N1-positive NSCLC undergoing inadequate versus no treatment (both $P<.001)$

\begin{tabular}{|c|c|c|c|c|c|c|}
\hline \multirow[b]{2}{*}{ Months } & \multicolumn{3}{|c|}{ No treatment } & \multicolumn{3}{|c|}{ Adequate treatment } \\
\hline & Mean \pm SD, $\%$ & $95 \% \mathrm{CI}$ & $\mathrm{n}$ at risk & Mean \pm SD, $\%$ & $95 \% \mathrm{CI}$ & $\mathrm{n}$ at risk \\
\hline 0 & 100 & & 2869 & 100 & & 4640 \\
\hline 12 & $37.90 \pm 1.00$ & $35.94-39.86$ & 929 & $55.70 \pm 0.70$ & $54.33-57.07$ & 2358 \\
\hline 24 & $19.40 \pm 0.80$ & $17.83-20.97$ & 401 & $28.80 \pm 0.74$ & $27.35-30.25$ & 1030 \\
\hline 36 & $12.10 \pm 0.70$ & $10.73-13.47$ & 214 & $17.00 \pm 0.60$ & $15.82-18.18$ & 524 \\
\hline 48 & $8.20 \pm 0.60$ & $7.02-9.38$ & 124 & $11.30 \pm 0.60$ & $10.12-12.48$ & 293 \\
\hline 60 & $6.70 \pm 0.60$ & $5.52-7.88$ & 87 & $7.70 \pm 0.50$ & $6.72-8.68$ & 159 \\
\hline 72 & $5.40 \pm 0.60$ & $4.22-6.58$ & 47 & $5.10 \pm 0.50$ & $4.12-6.08$ & 78 \\
\hline 84 & $4.40 \pm 0.60$ & $3.22-5.58$ & 25 & $4.20 \pm 0.40$ & $3.42-4.98$ & 46 \\
\hline 96 & NA & & & $2.50 \pm 0.50$ & $1.52-3.48$ & 11 \\
\hline
\end{tabular}

$S D$, Standard deviation; $C I$, confidence interval; $N A$, not applicable. 
TABLE E3. Ninety-five percent confidence intervals and number at risk periodically with time for Kaplan-Meier curve (shown in Figure 2) for overall survival in patients with clinical N1-positive NSCLC undergoing definitive CRT versus surgical resection $(P<.001)$

\begin{tabular}{|c|c|c|c|c|c|c|}
\hline \multirow[b]{2}{*}{ Months } & \multicolumn{3}{|c|}{ CRT } & \multicolumn{3}{|c|}{ Surgery } \\
\hline & Mean \pm SD, \% & $95 \%$ CI & $n$ at risk & Mean \pm SD, $\%$ & $95 \%$ CI & $n$ at risk \\
\hline 0 & $100 \%$ & & 3138 & 100 & & 9719 \\
\hline 12 & $72.30 \pm 0.80$ & $70.73-73.87$ & 2038 & $82.70 \pm 0.40$ & $81.92-83.48$ & 7014 \\
\hline 24 & $45.30 \pm 1.00$ & $43.34-47.26$ & 1043 & $66.10 \pm 0.50$ & $65.12-67.08$ & 4369 \\
\hline 36 & $31.40 \pm 1.00$ & $29.44-33.36$ & 561 & $54.00 \pm 0.60$ & $52.82-55.18$ & 2660 \\
\hline 48 & $22.90 \pm 0.90$ & $21.14-24.66$ & 295 & $45.50 \pm 0.70$ & $44.13-46.87$ & 1610 \\
\hline 60 & $15.90 \pm 1.00$ & $13.94-17.86$ & 137 & $38.40 \pm 0.70$ & $37.03-39.77$ & 956 \\
\hline 72 & $11.30 \pm 1.00$ & $9.34-13.26$ & 46 & $33.10 \pm 0.80$ & $31.53-34.67$ & 541 \\
\hline 84 & $8.10 \pm 1.30$ & $5.55-10.65$ & 11 & $27.80 \pm 0.90$ & 26.04-29.56 & 235 \\
\hline 96 & NA & & & $22.30 \pm 1.30$ & $19.75-24.85$ & 45 \\
\hline
\end{tabular}

CRT, Chemoradiation; $S D$, standard deviation; $C I$, confidence interval; $N A$, not applicable.

TABLE E4. Ninety-five percent confidence intervals and number at risk periodically with time for Kaplan-Meier curve (shown in Figure 3) for overall survival in $\mathbf{2 3 0 8}$ propensity score matched patient-pairs undergoing either surgical resection or definitive CRT for the treatment of N1 node-positive NSCLC $(P<.001)$

\begin{tabular}{|c|c|c|c|c|c|c|}
\hline \multirow[b]{2}{*}{ Months } & \multicolumn{3}{|c|}{ CRT } & \multicolumn{3}{|c|}{ Surgery } \\
\hline & Mean \pm SD, $\%$ & $95 \%$ CI & $\mathrm{n}$ at risk & Mean \pm SD, \% & $95 \%$ CI & $\mathrm{n}$ at risk \\
\hline 0 & 100 & & 2308 & 100 & & 2308 \\
\hline 12 & $73.50 \pm 0.90$ & $71.74-75.26$ & 1519 & $80.70 \pm 0.80$ & $79.13-82.27$ & 1637 \\
\hline 24 & $47.10 \pm 1.10$ & $44.94-49.26$ & 778 & $62.60 \pm 1.10$ & 60.44-64.76 & 990 \\
\hline 36 & $32.20 \pm 1.10$ & $30.04-34.36$ & 411 & $48.20 \pm 1.20$ & $45.85-50.55$ & 587 \\
\hline 48 & $23.90 \pm 1.10$ & $21.74-26.06$ & 214 & $40.50 \pm 1.30$ & $37.95-43.05$ & 337 \\
\hline 60 & $17.50 \pm 1.20$ & $15.15-19.85$ & 103 & $33.00 \pm 1.40$ & $30.26-35.74$ & 204 \\
\hline 72 & $12.30 \pm 1.20$ & $9.95-14.65$ & 33 & $27.60 \pm 1.50$ & $24.66-30.54$ & 106 \\
\hline 84 & $9.00 \pm 1.60$ & $5.86-12.14$ & 9 & $24.80 \pm 1.70$ & $21.47-28.13$ & 39 \\
\hline 96 & NA & & & $21.90 \pm 2.20$ & $17.59-26.21$ & 8 \\
\hline
\end{tabular}

CRT, Chemoradiation; $S D$, standard deviation; $C I$, confidence interval; $N A$, not applicable. 